ANNALES

POLONICI MATHEMATICI

$82.1(2003)$

\title{
On the Euler characteristic of the real Milnor fibres of an analytic function
}

\author{
by Piotr DudzińSKi (Gdańsk)
}

\begin{abstract}
The paper is concerned with the relations between real and complex topological invariants of germs of real-analytic functions. We give a formula for the Euler characteristic of the real Milnor fibres of a real-analytic germ in terms of the Milnor numbers of appropriate functions.
\end{abstract}

Introduction. In [3], McCrory and Parusiński proved that if $f: \mathbb{R}^{n}, 0$ $\rightarrow \mathbb{R}, 0$ is a germ of an analytic function, then the difference (and sum) of the Euler characteristics mod 4 of the real Milnor fibres of $f$ over $+\delta$ and $-\delta$ can be expressed in terms of the dimensions of the generalized eigenspaces of the algebraic monodromy.

In this paper we shall prove an analogous formula for $\mathcal{A}_{d^{-}}$-germs. The notion of an $\mathcal{A}_{d^{-}}$germ was introduced by Szafraniec in [5] as a generalization of a germ defined by a weighted homogeneous polynomial (the papers $[2,5]$ are concerned with topological invariants of $\mathcal{A}_{d^{-}}$-germs and generalize Wall's result [7]). In the case of $\mathcal{A}_{d^{-}}$germs, we obtain another description of the real Milnor fibres, without using the complex monodromy. Instead we prove that the sum of the Euler characteristics mod 4 of the real Milnor fibres over $\pm \delta$ can be expressed in terms of the Euler characteristics of the Milnor fibres of appropriate restrictions of $f_{\mathbb{C}}$, where $f_{\mathbb{C}}$ denotes the complexification of $f$ (Theorem 2). These characteristics, in turn, can be effectively calculated if 0 is an isolated critical point of $f_{\mathbb{C}}$, although the formula also holds in the non-isolated case.

In the first section we study the action of the dihedral group on the Milnor fibre of an analytic function. Theorem 1 describes the relation between the real and complex invariants of a real-analytic germ and it is the main tool we use in the proof of Theorem 2 .

2000 Mathematics Subject Classification: Primary 14P15.

Key words and phrases: Euler characteristic, Milnor fibre. 
1. Action of the dihedral group on the Milnor fibre of an analytic function. Let $d, w_{1}, \ldots, w_{n}$ be positive integers. For every $\lambda \in \mathbb{C}$ and $z=\left(z_{1}, \ldots, z_{n}\right) \in \mathbb{C}^{n}$ we shall write $\lambda . z=\left(\lambda^{w_{1}} z_{1}, \ldots, \lambda^{w_{n}} z_{n}\right)$. Write $d=p^{u} v$, where $p, u, v$ are positive integers such that $p$ is prime, $v$ is odd and prime to $p$. We may assume that $w_{k} \equiv 0 \bmod p$ if and only if $k \leq m=m(p)$ for some integer $m \leq n$.

Assume that $m<n$, i.e. some $w_{k}$ is not divisible by $p$. Set $\eta=\exp \left(\pi i / p^{u}\right)$ and $\varepsilon=\eta^{2}$. For $j=0,1, \ldots, p^{u}-1$ and $z \in \mathbb{C}^{n}$ we define $j(z)=\varepsilon^{j} . z=$ $\left(\varepsilon^{j w_{1}} z_{1}, \ldots, \varepsilon^{j w_{n}} z_{n}\right)$.

If $f: \mathbb{R}^{n}, 0 \rightarrow \mathbb{R}, 0$ is a germ of a real-analytic function, denote by $f_{\mathbb{C}}: \mathbb{C}^{n}, 0 \rightarrow \mathbb{C}, 0$ its complexification, by $F(f)$ the Milnor fibre of $f_{\mathbb{C}}$, and by $F_{\mathbb{R}}(f)$ the real Milnor fibre of $f$, i.e. $F(f)=f_{\mathbb{C}}^{-1}(\xi) \cap B_{r}^{2 n}$ and $F_{\mathbb{R}}(f)=f^{-1}(\xi) \cap B_{r}^{n}$, where $0<\xi \ll r \ll 1$ and $B_{r}^{2 n}$ (resp. $B_{r}^{n}$ ) denotes the ball of radius $r$ centred at the origin in $\mathbb{R}^{2 n}$ (resp. $\mathbb{R}^{n}$ ). Let $\tilde{f}$ denote the restriction of $f$ to $\mathbb{R}^{m} \times\{0\} \subset \mathbb{R}^{n}$. Recall that we do not assume that $f$ and $g$ have an isolated singularity at the origin.

Theorem 1. If $f, g: \mathbb{R}^{n}, 0 \rightarrow \mathbb{R}, 0$ are analytic such that

$$
f_{\mathbb{C}}\left(\varepsilon^{j} . z\right)=f_{\mathbb{C}}(z), \quad g_{\mathbb{C}}\left(\varepsilon^{j} . z\right)=g_{\mathbb{C}}(z)
$$

for $z \in \mathbb{C}^{n}, j \in \mathbb{Z}$, and

$$
f_{\mathbb{C}}\left(\eta^{j} \cdot x\right)= \begin{cases}f(x) & \text { if } j \text { is even } \\ g(x) & \text { if } j \text { is odd }\end{cases}
$$

for $x \in \mathbb{R}^{n}$ and $j \in \mathbb{Z}$, then

$$
\begin{aligned}
\chi(F(f))-\chi(F(\widetilde{f})) \equiv & a_{+} \chi\left(F_{\mathbb{R}}(f)\right)+a_{-} \chi\left(F_{\mathbb{R}}(g)\right) \\
& -p\left(\widetilde{a}_{+} \chi\left(F_{\mathbb{R}}(\widetilde{f})\right)+\widetilde{a}_{-} \chi\left(F_{\mathbb{R}}(\widetilde{g})\right)\right) \bmod 2 p^{u}
\end{aligned}
$$

where $a_{+} / a_{-}$(resp. $\left.\widetilde{a}_{+} / \widetilde{a}_{-}\right)$denote the number of even/odd integers $j$ such that $0 \leq j \leq p^{u}-1$ (resp. $\left.0 \leq j \leq p^{u-1}-1\right)$.

Set $a=p^{u}$. Condition (1) implies that the group $\mathbb{Z}_{a}$ acts on $F(f)$ (and on $F(g))$. Since $f$ is real-analytic, the complex conjugation also acts on $F(f)$. Let $G$ be the dihedral group of order $2 a$, i.e. the group generated by elements $\gamma, \beta$ with the relations $\gamma^{2}=1, \beta^{a}=1, \gamma \beta^{j}=\beta^{-j} \gamma, j \in \mathbb{Z}$. From the above, there is an action of $G$ on $F(f)$ given by $\gamma(z)=\bar{z}, \beta(z)=\varepsilon \cdot z$.

Define $A_{j}=\left\{z \in F(f) \mid \varepsilon^{j} . z=z\right\}$ for $j=0, \ldots, a-1$. Observe that if $j=a / p=p^{u-1}$, then $\varepsilon^{j w_{k}} z_{k}=z_{k} \exp \left(2 \pi w_{k} i / p\right)$; if $k>m$, that is, $w_{k}$ is not divisible by $p$, then $\exp \left(2 \pi w_{k} i / p\right) \neq 1$, and consequently $A_{a / p}=\{z \in$ $F(f) \mid z_{k}=0$ for $\left.k>m\right\}$.

LEMMA 1.

$$
\bigcup_{j=1}^{a-1} A_{j}=A_{a / p}
$$


Proof. Assume that $z \in F(f)$ and $z=\varepsilon^{j} . z$ for some $1 \leq j \leq a-1$. This means that $z_{k}=\varepsilon^{j w_{k}} z_{k}$ for $k=1, \ldots, n$. Assume that $z_{k} \neq 0$ for some $k$, i.e. $j w_{k}$ is divisible by $a=p^{u}$. Since $j$ is not divisible by $a$, it follows that $w_{k}$ is divisible by $p$. Hence $z_{k}=0$ for $k>m$ and $A_{j} \subset A_{a / p}$ for $1 \leq j \leq a-1$.

Lemma 2. (i) $\chi\left(B_{j}\right)= \begin{cases}\chi\left(F_{\mathbb{R}}(f)\right) & \text { if } j \text { is even, } \\ \chi\left(F_{\mathbb{R}}(g)\right) & \text { if } j \text { is odd. }\end{cases}$

(ii) If $0 \leq j<j^{\prime} \leq a-1$, then

$$
B_{j} \cap B_{j^{\prime}}=A_{a / p} \cap B_{j} \cap B_{j^{\prime}} .
$$

(iii) If $0 \leq j \leq a / p-1,0 \leq s \leq p-1$ and $j^{\prime}=j+s a / p$, then

$$
B_{j} \cap A_{a / p}=B_{j^{\prime}} \cap A_{a / p} \text {. }
$$

Proof. (i) Suppose that $\varepsilon^{j} \cdot \bar{z}=z$ for some $0 \leq j \leq a-1$. This means that $z_{k}=\varepsilon^{j w_{k}} \bar{z}_{k}, 1 \leq k \leq n$, hence $z_{k}^{2}=\varepsilon^{j w_{k}} z_{k} \bar{z}_{k}=\left(\eta^{j w_{k}}\left|z_{k}\right|\right)^{2}$. It follows that $z_{k}=\eta^{j w_{k}} x_{k}, x_{k} \in \mathbb{R}$. Set $x=\left(x_{1}, \ldots, x_{n}\right)$. Then, from condition (2),

$$
f_{\mathbb{C}}(z)= \begin{cases}f(x) & \text { if } j \text { is even, } \\ g(x) & \text { if } j \text { is odd }\end{cases}
$$

and consequently

$$
\chi\left(B_{j}\right)= \begin{cases}\chi\left(F_{\mathbb{R}}(f)\right) & \text { if } j \text { is even, } \\ \chi\left(F_{\mathbb{R}}(g)\right) & \text { if } j \text { is odd. }\end{cases}
$$

(ii) Assume that $z \in B_{j} \cap B_{j^{\prime}}$ and $k<m$. Then $z_{k}=\eta^{j w_{k}} x_{k}=\eta^{j^{\prime} w_{k}} x_{k}^{\prime}$ for some $x_{k}, x_{k}^{\prime} \in \mathbb{R}$. Clearly $\left|x_{k}\right|=\left|x_{k}^{\prime}\right|$. If $x_{k} \neq 0$, then $w_{k}\left(j^{\prime}-j\right)$ is divisible by $a=p^{u}$. Since $w_{k}$ is not divisible by $p$ for $k>m$, it follows that $j^{\prime}-j$ is divisible by $a$, which contradicts the assumption that $0 \leq j<j^{\prime} \leq a-1$.

(iii) Suppose that $z \in B_{j^{\prime}} \cap A_{a / p}$. Then $z_{k}=0$ for $k>m$ and $w_{k}$ is divisible by $p$ for $k \leq m$. Hence $z_{k}=\eta^{(j+s a / p) w_{k}} x_{k}= \pm \eta^{j w_{k}} x_{k}$, so $z \in$ $B_{j} \cap A_{a / p}$.

Proof of Theorem 1. The dihedral group $G$ of order $2 a$ acts freely on $F(f)-\bigcup_{j=0}^{a-1}\left(A_{j} \cup B_{j}\right)$, hence

$$
\chi(F(f)) \equiv \chi\left(\bigcup_{j=0}^{a-1}\left(A_{j} \cup B_{j}\right)\right) \bmod 2 a .
$$

According to Lemma $1, \bigcup_{j=1}^{a-1} A_{j}=A_{a / p}$. For simplicity we will write $B_{a}$ instead of $A_{a / p}$. Thus,

$$
\chi(F(f)) \equiv \chi\left(\bigcup_{j=0}^{a} B_{j}\right) \bmod 2 a .
$$


Clearly,

$$
\chi\left(\bigcup_{j=0}^{a} B_{j}\right)=\sum_{q=1}^{a+1}(-1)^{q-1} S_{q},
$$

where $S_{q}=\sum_{J} T_{J}, J=\left(j_{1}, \ldots, j_{q}\right), 0 \leq j_{1}<\ldots<j_{q} \leq a$ and $T_{J}=\chi\left(B_{j_{1}} \cap\right.$ $\left.\ldots \cap B_{j_{q}}\right)$. We may write $S_{q}=\sum_{H} T_{H}+\sum_{I} T_{I}$, where $H=\left(h_{1}, \ldots, h_{q}\right)$, $0 \leq h_{1}<\ldots<h_{q}=a, I=\left(i_{1}, \ldots, i_{q}\right), 0 \leq i_{1}<\ldots<i_{q}<a$.

Thus, we have $S_{q+1}=\sum_{I_{a}} T_{I_{a}}+\sum_{I^{\prime}} T_{I^{\prime}}$, where $I_{a}=\left(i_{1}, \ldots, i_{q}, a\right)$ and $I^{\prime}=\left(i_{1}^{\prime}, \ldots, i_{q+1}^{\prime}\right), 0 \leq i_{1}^{\prime}<\ldots<i_{q+1}^{\prime}<a$.

If $q=a$, then

$$
S_{q+1}=S_{a+1}=\chi\left(\bigcap_{j=0}^{a} B_{j}\right) .
$$

Due to Lemma 2(ii), $\sum_{I} T_{I}=\sum_{I_{a}} T_{I_{a}}$ and consequently

$$
\chi\left(\bigcup_{j=0}^{a} B_{j}\right)=\sum_{j=0}^{a} \chi\left(B_{j}\right)-\sum_{h=0}^{a-1} \chi\left(B_{h} \cap B_{a}\right) .
$$

Applying Lemma 2(i), (iii) we obtain

$$
\begin{aligned}
\chi\left(\bigcup_{j=0}^{a} B_{j}\right) & =a_{+} \chi\left(F_{\mathbb{R}}(f)\right)+a_{-} \chi\left(F_{\mathbb{R}}(g)\right)+\chi\left(B_{a}\right)-\sum_{j=0}^{a-1} \chi\left(B_{j} \cap B_{a}\right) \\
& =a_{+} \chi\left(F_{\mathbb{R}}(f)\right)+a_{-} \chi\left(F_{\mathbb{R}}(g)\right)+\chi\left(B_{a}\right)-p \sum_{j=0}^{a / p-1} \chi\left(B_{j} \cap B_{a}\right) .
\end{aligned}
$$

By the definition $\tilde{f}: \mathbb{R}^{m} \rightarrow \mathbb{R}$. For $z^{\prime}=\left(z_{1}, \ldots, z_{m}\right) \in \mathbb{C}^{m}$ and $j=0$, $1, \ldots, a / p-1$ we define $j\left(z^{\prime}\right)=\left(\varepsilon^{j w_{1}} z_{1}, \ldots, \varepsilon^{j w_{m}} z_{m}\right), \widetilde{B}_{j}=\left\{z^{\prime} \in F(\widetilde{f}) \mid\right.$ $\left.j\left(\bar{z}^{\prime}\right)=z^{\prime}\right\}, \widetilde{C}_{j}=\left\{z^{\prime} \in F(\widetilde{g}) \mid j\left(\bar{z}^{\prime}\right)=z^{\prime}\right\}$.

Using the same arguments as above one can prove that

$$
\chi\left(\widetilde{B}_{j}\right)= \begin{cases}\chi\left(F_{\mathbb{R}}(\widetilde{f})\right) & \text { if } j \text { is even, } \\ \chi\left(F_{\mathbb{R}}(\widetilde{g})\right) & \text { if } j \text { is odd. }\end{cases}
$$

Clearly, $\chi\left(B_{a}\right)=\chi(F(\widetilde{f}))$, and $\chi\left(\widetilde{B}_{j}\right)=\chi\left(B_{j} \cap B_{a}\right)$ for $j=0,1, \ldots, a / p-1$. Thus

$$
\begin{aligned}
\chi(F(f)) \equiv & a_{+} \chi\left(F_{\mathbb{R}}(f)\right)+a_{-} \chi\left(F_{\mathbb{R}}(g)\right)+\chi(F(\widetilde{f})) \\
& -p\left(\widetilde{a}_{+} \chi\left(F_{\mathbb{R}}(\widetilde{f})\right)+\widetilde{a}_{-} \chi\left(F_{\mathbb{R}}(\widetilde{g})\right)\right) \bmod 2 a .
\end{aligned}
$$

2. $\mathcal{A}_{d}$-germs. Let $f: \mathbb{R}^{n}, 0 \rightarrow \mathbb{R}, 0$ be a germ of a real-analytic function.

Definition. Let $d \geq 2$ be an integer. We shall say that $f$ is an $\mathcal{A}_{d^{-}}$germ if there are positive integers $w_{1}, \ldots, w_{n}$ such that if $f=\sum_{\alpha} a_{\alpha} x^{\alpha}$ and $a_{\alpha} \neq 0$ then $\alpha_{1} w_{1}+\ldots+\alpha_{n} w_{n} \equiv d \bmod 2 d$. 
EXAMPLES. (i) Each germ defined by a weighted homogeneous polynomial of degree $d$ is an $\mathcal{A}_{d^{-}}$germ.

(ii) The germ $f(x, y, z, t)=x^{4}+x^{12}+y^{2}+z^{3} t+z^{4} t^{4}$ is an $\mathcal{A}_{8}$-germ, where $w_{1}=2, w_{2}=4, w_{3}=1, w_{4}=5$.

Let $F_{+}$and $F_{-}$denote the positive and negative real Milnor fibres of $f$, that is, $F_{+}=f^{-1}(\delta) \cap B_{r}^{n}, F_{-}=f^{-1}(-\delta) \cap B_{r}^{n}$, where $0<\delta \ll r \ll 1$. Clearly, $F_{+}=F_{\mathbb{R}}(f)$ and $F_{-}=F_{\mathbb{R}}(-f)$. Let $\widetilde{F}_{+}$(resp. $\widetilde{F}_{-}$) denote the positive (resp. negative) real Milnor fibre of $\widetilde{f}$ (of course $\widetilde{f}$ is an $\mathcal{A}_{d}$-germ).

THEOREM 2. If $f$ is an $\mathcal{A}_{d}$-germ, then

$$
\left(\chi\left(F_{+}\right)+\chi\left(F_{-}\right)\right)-\left(\chi\left(\widetilde{F}_{+}\right)+\chi\left(\widetilde{F}_{-}\right)\right) \equiv 2(\chi(F(f))-\chi(F(\widetilde{f}))) / a \bmod 4 .
$$

Proof. We have $f_{\mathbb{C}}\left(\varepsilon^{j} . z\right)=f_{\mathbb{C}}(z)$ for $z \in \mathbb{C}^{n}, j \in \mathbb{Z}$. Moreover, $v$ is odd, hence if $x \in \mathbb{R}^{n}, j \in \mathbb{Z}$, then $f_{\mathbb{C}}\left(\eta^{j} . x\right)=(-1)^{j} f(x)$. This means that the germs $f$ and $-f$ satisfy conditions (1) and (2) of Theorem 1 . Thus, $\chi(F(f))-\chi(F(\widetilde{f})) \equiv a_{+} \chi\left(F_{+}\right)+a_{-} \chi\left(F_{-}\right)-p\left(\widetilde{a}_{+} \chi\left(\widetilde{F}_{+}\right)+\widetilde{a}_{-} \chi\left(\widetilde{F}_{-}\right)\right) \bmod 2 a$.

If $d$ is even then $p=2$ and $a_{+}=a_{-}=a / 2$. If $d$ is odd then the map $\left(x_{1}, \ldots, x_{n}\right) \mapsto\left((-1)^{w_{1}} x_{1}, \ldots,(-1)^{w_{n}} x_{n}\right)$ maps $F_{+}$homeomorphically onto $F_{-}$. Then $a \chi\left(F_{+}\right)=a \chi\left(F_{-}\right)=a\left(\chi\left(F_{+}\right)+\chi\left(F_{-}\right)\right) / 2($ similarly for $\widetilde{F})$. Hence in both cases we obtain

$\left.a\left(\chi\left(F_{+}\right)+\chi\left(F_{-}\right)\right) / 2-a\left(\chi\left(\widetilde{F}_{+}\right)+\chi\left(\widetilde{F}_{-}\right)\right) / 2 \equiv \chi(F(f))-\chi(F(\widetilde{f}))\right) \bmod 2 a$. It follows that

$$
\left(\chi\left(F_{+}\right)+\chi\left(F_{-}\right)\right)-\left(\chi\left(\widetilde{F}_{+}\right)+\chi\left(\widetilde{F}_{-}\right)\right) \equiv 2(\chi(F(f))-\chi(F(\widetilde{f}))) / a \bmod 4 .
$$

As mentioned above, $\tilde{f}$ is an $\mathcal{A}_{d}$-germ, so Theorem 2 may be applied to $\widetilde{f}$, and so on. Repeated application of Theorem 2 enables us to express the number $\chi\left(F_{+}\right)+\chi\left(F_{-}\right) \bmod 4$ only in terms of the Euler characteristics of the Milnor fibres of appropriate restrictions (given by the weights $w_{i}$ ) of $f_{\mathbb{C}}$. In the case of an algebraically isolated singularity of $f$, i.e., when $0 \in \mathbb{C}^{n}$ is isolated in the set of critical points of $f_{\mathbb{C}}$, those characteristics can be calculated effectively from the Milnor numbers of $f_{\mathbb{C}}, \widetilde{f}_{\mathbb{C}}$, etc. Recall that the Milnor number of $f_{\mathbb{C}}$ equals the dimension of an appropriate local algebra $([4])$. Moreover, if $f_{\mathbb{C}}$ has an isolated singularity, then also $\widetilde{f}_{\mathbb{C}}$ has one $([2])$. When 0 is not an isolated critical point of $f_{\mathbb{C}}$, then one can use Varchenko's method ([6]), although it is less effective.

\section{References}

[1] A. Dimca and L. Paunescu, Real singularities and dihedral representations, Workshop on Real and Complex Singularities (São Carlos, 1996), Math. Contemp. 12 (1997), 67-82. 
[2] P. Dudziński, Topological invariants of germs of real-analytic functions, Glasgow Math. J. 39 (1997), 85-89.

[3] C. McCrory and A. Parusiński, Complex monodromy and the topology of real algebraic sets, Compositio Math. 106 (1997), 211-233.

[4] J. Milnor, Singular Points of Complex Hypersurfaces, Princeton Univ. Press, 1968.

[5] Z. Szafraniec, On the topological invariants of germs of analytic functions, Topology 26 (1987), 235-238.

[6] A. N. Varchenko, Zeta-function of monodromy and Newton's diagram, Invent. Math. 37 (1976), 253-262.

[7] C. T. C. Wall, Topological invariance of the Milnor number mod 2, Topology 22 (1983), 345-350.

Institute of Mathematics

University of Gdańsk

Wita Stwosza 57

80-925 Gdańsk, Poland

E-mail: pd@delta.math.univ.gda.pl

Reçu par la Rédaction le 24.4.2002

Révisé le 24.2.2003 